\title{
ÍNDICES DE CONFORTO NA AVALIAÇÃO DO BEM ESTAR ANIMAL DE MATRIZES SUÍNAS EM DIFERENTES SISTEMAS DE CRIAÇÃO
}

\author{
VIEIRA, Renata de Fátima Nogueira ${ }^{1}$ \\ SILVA, Késia Oliveira ${ }^{2}$ \\ MELLO, Silvio de Paula ${ }^{3}$ \\ MARTINS, José Reinaldo ${ }^{4}$
}

Recebido em: 2010-02-21

Aprovado em:2010-03-27

ISSUE DOI: $10.3738 / 1982.2278-367$

RESUMO: O objetivo do trabalho foi avaliar o bem estar animal de matrizes suínas em diferentes sistemas de criação. O experimento foi conduzido no Setor de Suinocultura do Sítio das Acácias da Faculdade Dr. Francisco Maeda, localizada no município de Ituverava-SP. Foram selecionadas quatro matrizes com as mesmas características fisiológicas (número de partos, idade e raça) e mesma data do parto. Duas matrizes foram dispostas em baias individuais com gaiola e outras duas colocadas em baias sem gaiola, com cama sobreposta (maravalha). O delineamento experimental adotado para análise foi o inteiramente ao acaso (DIC) adotando-se como tratamentos os sistemas de alojamento em baias com gaiola e baias sem gaiola com cama sobreposta, com duas porcas em cada tratamento. Os índices ambientais e fisiológicos, independente do sistema de alojamento, apresentaram-se próximos ou além da zona de conforto para matrizes em lactação.

Palavras-chave: Suíno. Bem-estar. Cama-sobreposta

SUMMARY: The purpose of this work was to evaluate the comfort of the womb's swine in different systems of breeding. The experiment was leaded at the Swine's Sector in the "Acácias" ranch of the college Dr. Francisco Maeda, situated in the city council of Ituverava-Sp. Four wombs were selected with the same physiological characters (numbers of parturition, age and descent) and the same parturition's date. Two wombs were prepared in individual stall with cage and the other two in stall without cage, with deep bedding. The experimental drawing was a completely randomized block and the system of shelter in stall with cages and stall without cage with deep bedding, with two pigs each were the treatments used. Independently of the shelter system, the environment and physiological registers showed next or beyond the comfort zone for the wombs in lactation.

Key word: Swine. Welfare. Deep bedding.

\section{INTRODUÇÃO}

Atualmente, com a exigência do mercado em que cada vez mais as pessoas desejam comer carne oriunda de animais que foram criados, tratados e abatidos em sistemas que promoveram seu bem-estar, baixou-se a padronização do bem-estar animal, inclusive com regulamentações e normas. A ausência do bem-estar repercute diretamente na sanidade dos alimentos, neste caso, a carne suína.

\footnotetext{
${ }^{1}$ Engenheira Agrônoma da FE/FAFRAM - email: renatanvieira@ hotmail.com

${ }^{2}$ Professora Dra. da ESALQ Dep. Eng Rural: kosilva@esalq.usp.br

${ }^{3}$ Professor Dr. da FE/FAFRAM - email: spmello@feituverava.com.br

${ }^{4}$ Técnico em agropecuária e funcionário do setor de Suinocultura da FAFRAM - email: j.rmartins@ hotmail.com
} 
Segundo a FAO, o crescimento anual de consumo de carnes no mundo até o ano 2015 deve ficar em torno de $2 \%$. Considerando ser a carne suína a mais produzida no mundo, uma parcela significativa deste percentual deverá ser atendida via expansão da produção de suínos. A posição dos principais países produtores de carne suína (China, União Européia e Estados Unidos) não deve ser alterada pelo menos no curto e médio prazos.

Com base na análise dos problemas e potencialidades dos grandes produtores mundiais, fica claro que o Brasil apresenta amplas possibilidades de se firmar como grande fornecedor de proteína animal. Estudos recentes mostram que o Brasil apresenta o menor custo de produção mundial, cerca de US $\$ 0,55 / \mathrm{kg}$, e produz carcaças de qualidade comparada à dos grandes exportadores. Dessa forma, pode-se dizer que o mercado internacional sinaliza para o crescimento das exportações brasileiras, com possibilidades de abertura de novos mercados como o do NAFTA, China, África do Sul, Chile e Taiwan. A abertura do Mercado Europeu para a carne suína brasileira deverá merecer atenção especial, assim como também o ingresso no Japão que é o maior importador mundial.

Além da produtividade e competitividade econômica, qualquer sistema de produção deve primar pela proteção ambiental, não somente pela exigência legal, mas, também, por proporcionar melhor qualidade de vida à população rural e urbana. Com relação à proteção ambiental, o produtor deve implantar um sistema de gestão ambiental integrado, contemplando as seguintes etapas: Avaliação do impacto ambiental, Manejo voltado para a proteção ambiental, Manejo nutricional e Manejo de água na propriedade.

O tipo ideal de edificação deve ser definido, fazendo-se um estudo detalhado do clima da região e (ou) do local onde será implantada a exploração, determinando as mais altas e baixas temperaturas ocorridas, a umidade do ar, a direção e a intensidade do vento. Assim, é possível projetar instalações com características construtivas capazes de minimizar os efeitos adversos do clima sobre os suínos.

Os consumidores, cada vez mais exigentes, estão dando maior ênfase ao bem-estar animal e à qualidade da carne, da concepção ao abate. Desta forma, o tema bem-estar animal vem de "fora para dentro“, ou seja, se expressa da sociedade para a atividade de produção (indústria) e tem se apresentado cada vez mais nas preocupações morais das pessoas desde as últimas décadas do século passado. Portanto, melhorar o bem-estar animal é necessário em nosso país, principalmente para atender às exigências do mercado consumidor, seja ele interno ou externo, que está cobrando dos produtores e técnicos uma produção de alimentos com menor agressão ao meio ambiente e respeito aos princípios de bem-estar animal (DALLA COSTA et al., 2008). 
No manejo de suínos, há fatores estressantes que, em função da duração ou severidade, são capazes de alterar a qualidade da carne e o bem-estar animal. Assim, dependendo da forma de condução dos suínos, pode-se observar que maus tratos, medo, esforço e excessiva utilização de choque elétrico que resultam em perdas econômicas a produtores e indústrias, em função da redução da qualidade da carne (DALLA COSTA et al., 2008).

O ambiente térmico no interior das instalações para suínos tem importância vital para o sucesso da atividade suinícula. Esse ambiente pode ser representado pelo índice de temperatura de globo e umidade (ITGU), que inclui a temperatura, a umidade relativa e a velocidade do ar, e a radiação.

A temperatura ambiente pode influenciar a produtividade dos suínos, por alterar a troca de calor com o ambiente, a taxa de ingestão de alimento e o ganho de peso e, concomitantemente, afetar ou mudar as exigências em nutrientes dos animais.

De acordo com Perdomo (1994), a reação do suíno ao estresse por calor pode ser constatada do ponto de vista fisiológico e metabólico, pelas alterações na temperatura corporal, pelos movimentos respiratórios e cardíacos, bem como pela mobilização de gordura corporal dos animais, e do ponto de vista da produtividade, por redução no consumo voluntário de ração e perda de peso. Fatores ambientais externos podem interferir no microclima gerado no interior das instalações, causando com isso impacto sobre a produção, com conseqüentes prejuízos econômicos à exploração.

Uma forma de avaliar as respostas dos animais ao ambiente térmico é por meio da observação de alguns parâmetros fisiológicos, como a temperatura retal (TR) e a freqüência respiratória (FR).

A medida da temperatura retal orienta a determinação do equilíbrio entre o ganho e a perda de calor do corpo, sendo essa medida usada freqüentemente como índice de adaptabilidade (MOTA, 1997). Para HAHN et al. (1997), o aumento da FR, quando considerado por curto período, é um mecanismo eficiente de perda de calor. Porém, quando os valores ultrapassam 120 movimentos respiratórios por minuto (mov min-1), o animal está sofrendo com a carga excessiva de calor e, acima de 160mov min-1, as medidas de emergência devem ser tomadas a fim de amenizar o estresse.

As variações da TR e da FR podem ser influenciadas, tanto por fatores intrínsecos (idade, raça, estado fisiológico), quanto por fatores extrínsecos (hora do dia, ingestão de alimentos e de água, temperatura ambiente, velocidade do vento, estação do ano). O presente trabalho teve como objetivo avaliar a vocalização e o bem estar animal de matrizes suínas em diferentes sistemas de criação. 


\section{MATERIAL E MÉTODOS}

O experimento foi conduzido no Setor de Suinocultura do Sítio das Acácias da Faculdade Dr. Francisco Maeda, localizada no município de Ituverava-SP (20²0’30’' S e 47²4’30' W e 690 metros) com temperatura média anual de $25,5^{\circ}$ C. Foram selecionadas quatro matrizes com as mesmas características fisiológicas (número de partos, idade e raça) e mesma data do parto. Duas matrizes foram dispostas em baias individuais com gaiola e outras duas colocadas em baias sem gaiola, com cama sobreposta (maravalha). As matrizes ficaram dispostas individualmente com seus respectivos leitões, no galpão de maternidade. Os leitões permaneceram dentro do escamoteador ou fora com suas respectivas mães. Foram quatorze dias de coleta, acompanhando o desmame dos leitões da granja. Durante esse período foram coletados as variáveis ambientais e índices fisiológicos diariamente em horários préestabelecidos, sendo às (7:00 hs; 8:00 hs e 9:00 hs), às (11:00hs;12:00 hs e 13:00 hs) e (15:00 hs;16:00 e17:00hs). Para confirmar o nível de conforto e estresse das matrizes nas duas instalações, foram avaliadas variáveis ambientais (Temperatura de bulbo seco, temperatura de globo negro, umidade relativa, ITGU e Entalpia), índices fisiológicos (temperatura retal e frequiência respiratória), hormônios (Cortisol, T3 e T4). O delineamento experimental adotado para análise foi o inteiramente ao acaso (DIC) adotando-se como tratamentos os sistemas de alojamento em baias com gaiola e baias sem gaiola com cama sobreposta, com duas porcas em cada tratamento. As variáveis meteorológicas e ambientais e os parâmetros fisiológicos foram analisados sendo utilizado o teste de Tukey para a comparação entre as médias, utilizando o programa estatístico Statistical Analysis System (SAS, 1992).

\section{RESULTADOS E DISCUSSÃO}

Na Tabela 1 são evidenciados os resultados médios das variáveis ambientais nos sistemas de alojamento avaliados. Os sistemas de alojamento apresentaram diferenças significativas $(\mathrm{P}<0,01)$ em todas as variáveis ambientais. $\mathrm{O}$ sistema de alojamento em baia sem gaiola (BSG) apresentou as maiores médias para Tbs $\left(22,31{ }^{\circ} \mathrm{C}\right)$, UR ( $\left.52,55 \%\right)$, Tbn $\left(22,63{ }^{\circ} \mathrm{C}\right)$, ITGU $(68,10)$ e H $(59,83$ kJ.kg-1) em relação ao sistema de alojamento em baia com gaiola (BCG). 
Tabela 1. Variáveis ambientais para o sistema de alojamento em baias individuais com gaiola (BCG) e sem gaiola (BSG). Environmental variables for the system of housing in individual stalls with cage (BCG) and without cage (BSG).

\begin{tabular}{lccccc}
\hline Sistema de alojamento & Tbs $\left({ }^{\circ} \mathrm{C}\right)$ & UR $(\%)$ & Tbn $\left({ }^{\circ} \mathrm{C}\right)$ & ITGU & H $\left(\mathrm{kJ}^{\mathrm{kg}}{ }^{-1}\right)$ \\
\hline BCG & $20,06 \mathrm{~b}$ & $48,58 \mathrm{~b}$ & $20,45 \mathrm{~b}$ & $64,79 \mathrm{~b}$ & $55,74 \mathrm{~b}$ \\
BSG & $22,31 \mathrm{a}$ & $52,55 \mathrm{a}$ & $22,63 \mathrm{a}$ & $68,10 \mathrm{a}$ & $59,83 \mathrm{a}$
\end{tabular}

Medias com letras diferentes, na mesma coluna, diferem $(\mathrm{P}<0,01)$ pelo teste de Tukey.

Tbs= Temperatura de bulbo seco; UR= Umidade relativa; Tbn= Temperatura de bulbo negro; ITGU = índice de temperatura de globo e umidade; $\mathrm{H}=$ Entalpia.

Há evidência do efeito da temperatura nos dois sistemas de alojamento e que nessas condições verifica-se que o condicionamento térmico no interior das duas instalações esteve próximo da temperatura máxima considerada ótima para a matriz, que varia de 7 a $23^{\circ} \mathrm{C}$ (NOBLET et al., 1989), verificando que os animais nessas condições podem ter sua reprodução comprometida, principalmente em fêmeas primíparas (LOVE et al., 1995; PELTONIEMI et al., 1999; NUNES et al., 2003). Observou-se que, durante o estudo, as temperaturas médias mantiveram-se próximas à faixa de conforto dos animais, entre 20,06 e 22,31 ${ }^{\circ} \mathrm{C}$. No entanto, a temperatura manteve-se, durante toda a fase avaliada, abaixo da temperatura crítica superior (TCS), não atingindo, em média, 28 C (MOUNT, 1968; Curtis, 1983; Silva, 1999), promovendo condições inadequadas, em que os animais submetidos a esses ambientes passariam a sofrer estresse calórico e reflexo direto na manutenção dos processos reprodutivos. Os valores médios de UR observados para os tratamentos, ficaram situados numa faixa abaixo do valor máximo, recomendada por Esmay (1982) e Nääs et al. (1995), de 70\%. No sistema de alojamento de baia sem gaiola com cama sobreposta, os valores médios para temperatura da cama, umidade relativa e temperatura do ambiente, foram de 25,62oC, 50,67\% e 26,43\%, respectivamente. Esses valores obtidos nesse microclima, formado pela cama sobreposta e animal podem ter influenciado os maiores valores de ITGU e entalpia, levando o animal a um estresse e ausência de bem estar. O piso de cama para suínos em crescimento pode influenciar a temperatura ambiente. Segundo Matte (1993), a maioria dos efeitos positivos de instalações com sistema de cama em baias abertas em comparação a pisos de concreto ocorre em condições de frio (abaixo de $10{ }^{\circ} \mathrm{C}$ ).

A Tabela 2 apresenta as médias das variáveis fisiológicas dos animais, a saber, temperatura retal $\left({ }^{\circ} \mathrm{C}\right)$ e freqüência respiratória (mov.min.-1), obtidas diariamente, durante o período experimental. Verifica-se uma diferença significativa $(\mathrm{P}<0,01)$ nas variáveis fisiológicas nos dois sistemas de alojamento. Com relação à temperatura retal, notam-se diferenças significativas entre os sistemas de alojamento, apresentando valores de $37,65^{\circ} \mathrm{C}$ 
(BCG) e $38,30{ }^{\circ} \mathrm{C}$ (BSG). Porém, para ambos os tratamentos, a variação da temperatura retal encontra-se próxima à condição de conforto, entre $38,6{ }^{\circ} \mathrm{C}$ e $39,3{ }^{\circ} \mathrm{C}$.

Tabela 2. Variáveis fisiológicas para o sistema de alojamento em baias individuais com gaiola (BCG) e sem gaiola (BSG). Physiological variables to the system of housing in individual stalls with cage (BCG) and without cage (BSG).

\begin{tabular}{lcc}
\hline Sistema de alojamento & TR $\left({ }^{\circ} \mathrm{C}\right)$ & FR (mov.min..$\left.^{-1}\right)$ \\
\hline BCG & $37,65 \mathrm{~b}$ & $26,48 \mathrm{~b}$ \\
BSG & $38,30 \mathrm{a}$ & $35,73 \mathrm{a}$ \\
\hline
\end{tabular}

Medias com letras diferentes, na mesma coluna, diferem $(\mathrm{P}<0,01)$ pelo teste de Tukey.

TR=Temperatura Retal; FR=Freqüência Respiratória.

A frequência respiratória apresentou diferença estatística entre os tratamentos BCG e BSG, com valores da ordem de 26,48 mov.min.-1 e 35,73 mov.min.-1 respectivamente, mostrando-se acima da condição de conforto para animais dessa categoria. Fêmeas em lactação em condição de conforto térmico apresentam freqüência respiratória em torno de 20 a 30 mov.min.-1 (MUIRHEAD; ALEXANDER, 1997). Os movimentos respiratórios podem aumentar de 22 a 100\%, em função do ambiente térmico, de acordo com Hannas et al. (1999).

Nas figuras a seguir são apresentados os valores de entalpia, temperatura retal e frequiência respiratória nos dois sistemas de alojamento BCG com piso de concreto e BSG com cama sobreposta.

A Figura 1 evidencia os valores de menor entalpia nos diferentes sistemas de criação das matrizes, apresentando menores valores no período da manhã (8:00 às11:00 h.), aumentando às 12:00 h. e começando a decrescer no período da tarde (16:00 às 17:00h.). A temperatura retal permaneceu próxima de $40^{\circ} \mathrm{C}$ durante todo o dia, já para a frequiência respiratória houve uma maior taxa respiratória no período da tarde.

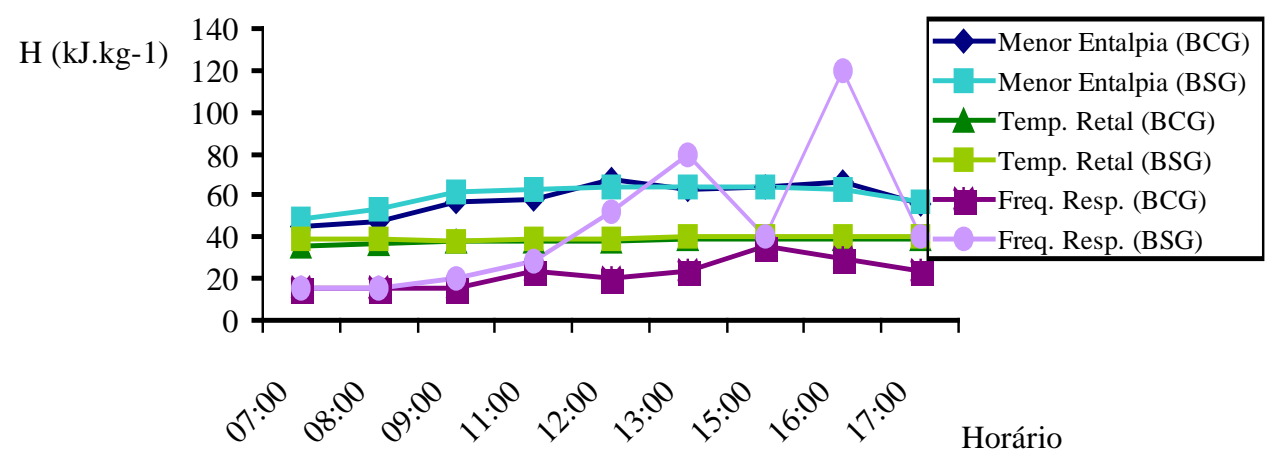

Figura 1. Valores da menor entalpia, temperatura retal e frequência respiratória da BCG e BSG. 


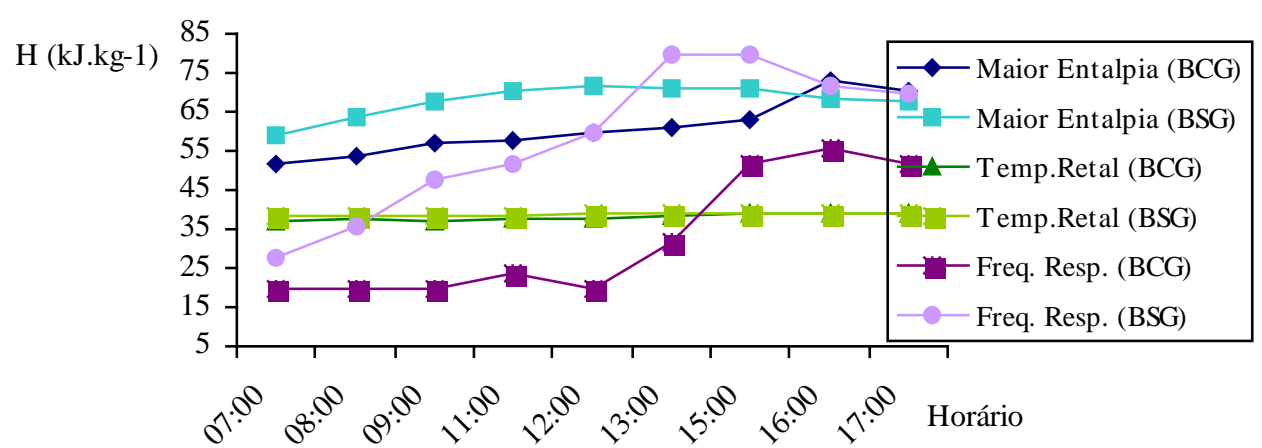

Figura 2. Valores da maior entalpia, temperatura retal e frequência respiratória na BCG e BSG.

A Figura 2 mostra os valores de maior entalpia nas BCG e BSG, verificando um aumento durante o período; verificado também na freqüência respiratória. A temperatura retal também permaneceu próxima de $40^{\circ} \mathrm{C}$ durante todo o período. Os valores encontrados para entalpia nas Figuras 13 e 16 estão dentro da zona de conforto recomendado para matrizes em lactação, não excedendo o valor limite para este índice (73,80 kJ.kg-1) (SILVA, 1999). Já para a temperatura retal, os valores estão próximos do limite máximo recomendado para essa categoria animal.

\section{CONCLUSÃO}

De acordo com os índices ambientais e fisiológicos, independente do sistema de alojamento, os valores apresentaram-se próximos da zona de conforto para matrizes em lactação.

\section{REFERÊNCIAS}

CURTIS, S.E. Environmental manegement in animal agriculture. Ames: State University Press, 1983. 409p.

DALLA COSTA, O. A. et al. Técnicas de manejo racional no desembarque de suínos destinados ao abate. Embrapa, CNPSA,2008. (Comunicado Técnico).

ESMAY, M.L. Principles of animal environment. West Port CT: ABI, 1982. 325p.

HAHN, G.L. et al. Cattle respiration rate as a function of ambient temperature. Transactions of American Society of Agricultural Engineering, v.40, p.97-121, 1997. 
HANNAS, M.I. et al. Efeito da temperatura ambiente sobre os parâmetros fisiológicos e hormonais de leitões dos 15 aos 30 kg. In: REUNIÃO ANUAL DA SOCIEDADE BRASILEIRA DE ZOOTECNIA, 36., 1999, Porto Alegre. Anais... Porto Alegre: SBZ, 1999. 1 CD-ROM.

LOVE, R.J.; KLUPIEC, C.; THORNTON, E.J. et al. An interaction between feeding rate and season affects fertility of sows. Animal Reproduction Science, v.39, p.275-284, 1995.

MATTE, J.J. A note on the effect of deep-litter housing on the growth performance of growing finishing pigs. Canadian Journal of Animal Science, v.73, p.642-647, 1993.

MOTA, L.S. Adaptação e interação genótipo-ambiente em vacas leiteiras. 1997. 69f. Tese (Doutorado em Ciências). Curso de Pós-graduação em Biologia Comparada. Universidade de São Paulo. Faculdade de Medicina de Ribeirão Preto.

MOUNT, L.E. The climate physiology of the pig. Baltimore: Williams and Welkins, 1968. 271p.

MUIRHEAD, M.; ALEXANDER, T. Managing pig health and the treatement of disease. Sheffield: 5Mentreprises, 1997. 608p.

NÄÄS, I.A. Estado da arte de bioclimatologia e necessidade de pesquisas futuras no Brasil. In: REUNIÃO ANUAL DA SOCIEDADE BRASILEIRA DE ZOOTECNIA, 31, 1995, Brasília, DF. Anais... Brasília: SBZ, 1995, p. 133-135.

NOBLET, J. et al. Effect of ambient temperature and adition of straw or alfafa in the diet on energy metabolism in pregnant sows. Livestock Production Science. v.21, p.309-324. 1989.

NUNES, C.G.V.; COSTA, E.P.; OLIVEIRA, R.F.M. et al. Efeito do acondicionamento térmico ambiental sobre o desempenho reprodutivo da fêmea suína. Revista Brasileira de Zootecnia, v.32, n.4, p.854-863, 2003.

PELTONIEMI, O.A.T. et al. Seasonal and management effects on fertility of the sow: a descriptive study. Animal Reproduction Science, v.55, p.47-61, 1999.

SILVA, I.J.O. Sistemas naturais e artificiais do controle do ambiente - climatização. In: SILVA, I.J.O. (Ed.) Ambiência e qualidade na produção industrial de suínos. Piracicaba: Fundação de Estudos Agrários Luiz de Queiroz, 1999. p.81-101.

STATISTICAL ANALYSIS SYSTEM - SAS. Statistical Analysis System: realease 6.08 (software). Cary: SAS Institute, 1992. 620p. 